\title{
Optimierte IgG-Antikörperdiagnostik der Bettfedern-Alveolitis mittels EAA-Suchtest
}

\section{Improved IgG Antibody Diagnostics of Feather Duvet Lung by an Antibody Screening Test}

Autoren

Institut
J. Sennekamp, E. Lehmann

Allergologisch-Immunologisches Labor, Malteser Lungen- und Allergiezentrum, Bonn eingereicht $\quad 7.6 .2015$ akzeptiert nach Revision 13.8.2015

Bibliografie

Dol http://dx.doi.org/ 10.1055/s-0034-1393065 Online-Publikation: 12.10.2015 Pneumologie 2015; 69: 638-644 (c) Georg Thieme Verlag KC Stuttgart · New York ISSN 0934-8387

Korrespondenzadresse Prof. Dr. Joachim Sennekamp cand. med. Emilia Lehmann Malteser Lungen- und Allergiezentrum Bonn Allergologisch-Immunologisches Labor

Weberstr. 118

53113 Bonn

sennekamp@t-online.de

\section{Zusammenfassung \\ $\nabla$}

Die heute zu selten diagnostizierte BettfedernAlveolitis kann häufiger erkannt werden, wenn die Analyse der IgG-Antikörper gegen Entenund Gänsefedern in den üblichen EAA-Suchtest integriert wird. Der Suchtest muss dafür nicht um die Antigene Enten- und Gänsefedern erweitert werden. Denn die Auswertung von 100 Seren, die in dieser Studie auf Tauben-, Wellensittich-, Enten- und Gänsefedern untersucht wurden, zeigt, dass eindeutig positive BettfedernAntikörper nicht isoliert, sondern nur zusammen mit Tauben- und Wellensittich-Antikörpern vorkommen. Somit eignen sich die Tauben- und Wellensittich-Antikörper zum Erkennen der Bettfedern-Antikörper. Seren ohne Tauben- und Wellensittich-Antikörper enthalten auch keine eindeutig positiven Bettfedern-Antikörper. Überdies stellte sich heraus, dass Seren mit stark positiven Tauben- und Wellensittich-Antikörpern immer gleichzeitig auch Bettfedern-Antikörper enthalten. Unsere Auswertung zeigt, dass 71\% der Bettfedern-Antikörperbestimmungen ohne wesentlichen Qualitätsverlust entbehrlich sind, wenn die Tauben- und Wellensittich-Antikörper des EAASuchtests in einem Zwei-Stufen-Test mit berücksichtigt werden.

\section{Einleitung und Fragestellung \\ $\nabla$}

In den letzten Jahren gewinnt die exogen-allergische Alveolitis (EAA) von Enten- und Gänsedaunenfedern, die Bettfedern-Alveolitis, zunehmend an Bedeutung [1-5]. Großes Aufsehen erregt aktuell eine Studie von Morell aus Barcelona, der 20 von 46 leitliniengerecht diagnostizierte idiopathische Lungenfibrosen als chronische allergische Alveolitiden entlarvte [5-6]. Die EAA diagnostizierte er mit dem vollen Arsenal der EAA-Diagnostik einschließlich Hauttest, chirurgischen Lungenbiopsien und inhalativen Provokationstests.

\section{Abstract \\ $\checkmark$}

The underdiagnosed feather duvet lung, an extrinsic allergic alveolitis (hypersensitivity pneumonitis) caused by duck and goose feathers, can be more frequently diagnosed, if duck and goose feather antibodies are included in the panel of the routinely applied IgG antibody screening test. This does not necessarily require extending the screening test to include duck and goose feather antigens. By analysing 100 sera with duck and goose antibodies we found that the commonly used pigeon and budgerigar antibodies can also screen for feather duvet antibodies. All examined sera lacking pigeon and budgerigar antibodies also lacked clear-cut duck and goose feather antibodies. The examined sera with strong pigeon or budgerigar antibodies always also contained feather duvet antibodies. However, sera with medium or low concentrated pigeon or budgerigar antibodies are not always associated with feather duvet antibodies. In the light of these observations, we find that $71 \%$ of the duck and goose antibody analyses would be dispensable without essential loss of quality, if the results of screening for pigeon and budgerigar antibodies were incorporated into the procedure of a step-by- step diagnostics.

Dabei stellte sich heraus, dass bei der Hälfte dieser Patienten (bei 10 von 20) keine idiopathische Lungenfibrose (IPF), sondern eine BettfedernAlveolitis vorlag.

Bisher galt die Bettfedern-Alveolitis als ausgesprochene Rarität. Zunächst nur beim Rupfen von Enten und Gänsen sowie beim Sortieren von Bettfedern bei wenigen Personen beobachtet [79], wurde erst ab den achtziger Jahren erkannt, dass Schlafen in Federbetten schon ausreicht, erkranken zu können $[1,10]$. Dies wird als Bettfedern-Alveolitis bezeichnet [11]. Als Antigenreservoire kommen auch Winterjacken, Sofakissen und 


\begin{tabular}{|lllll|}
\hline $\begin{array}{l}\text { IgG-Antikörper } \\
\text { in } \mathbf{m g A} / \mathbf{I}\end{array}$ & Entenfedern & Gänsefedern & $\begin{array}{l}\text { Taube } \\
\text { Serum-, Federn-, Kot-Mix }\end{array}$ & $\begin{array}{l}\text { Wellensittich } \\
\text { Serum-, Federn-, Kot-Mix }\end{array}$ \\
\hline fraglich positiv & $9-10$ & $11-14$ & $16-17$ & $7-11$ \\
\hline schwach positiv & $11-27$ & $15-38$ & $18-26,5$ & $12-39$ \\
\hline mittelstark positiv & $28-74,5$ & $39-124,5$ & $27-134$ & $40-200$ \\
\hline stark positiv & $\geq 75$ & $\geq 125$ & $\geq 135$ & $\geq 201$ \\
\hline
\end{tabular}

Tab. 1 Bereiche der IgG-Antikörper gegen Vogel-Antigene (aus [16]).
Polstermöbel in Betracht. Da somit Enten- und Gänsefedern sehr weit verbreitet sind, lässt sich ihr Vorkommen bei der Anamneseerhebung nicht so einfach erfragen wie der Kontakt mit einem Ziervogel oder eine Farmerlunge bei Arbeiten mit schimmligem Heu. Deshalb hat die Antikörperbestimmung für die Diagnostik der Bettfedern-Alveolitis eine besonders wichtige Funktion. Nach unserer Erfahrung wird bei Vorliegen einer EAA häufig nicht an eine Allergie gegen Enten- und Gänsefedern gedacht. Deshalb machen wir uns Gedanken, wie die serologische Antikörperdiagnostik der Bettfedern-Alveolitis ohne große zusätzliche Kosten verbessert werden kann.

Bei Patienten mit Hinweisen auf eine EAA, aber leerer Anamnese auf ein verdächtiges Antigen, wird oft ein IgG-Antikörper-Suchtest auf die häufigsten EAA-Antigene (EAA-Suchtest, EAA-Screening-Test) durchgeführt [12]. Dieser Screening-Test enthält bei uns die Federn von Taube und Wellensittich, Thermoactinomyceten (Saccharopolyspora rectivirgula und Thermoactinomyces vulgaris) und die Schimmelpilze Aspergillus fumigatus, Penicillium chrysogenum, Aureobasidium pullulans und Cephalosporium acremonium (Acremonium kiliense), aber nicht die Bettfedern von Ente und Gans. Andere Labors setzten ähnliche Suchtests ohne Bettfedern ein.

Da die Vogelfedern-Antigene untereinander verwandt sind [13, 14] und wir im Laufe der letzten Jahre den Eindruck gewonnen haben, dass Antikörper gegen Taube und Wellensittich häufiger und stärker positiv vorkommen als Antikörper gegen Bettfedern, soll in diesem Beitrag geprüft werden, ob die Antikörpertests auf Taube und Wellensittich die Bettfedern-Antikörper miterfassen. Dann brauchten nur noch die Seren, die auf Taube und Wellensittich positiv reagieren, in einem zweiten Ansatz auf Enten- und Gänsefedern untersucht werden. Dies würde eine Erleichterung und Kostenersparnis der serologischen Diagnostik der Bettfedern-Alveolitis bedeuten.

Eine weitere Vereinfachung der serologischen Diagnostik könnte erfolgen, wenn sich unser Eindruck bestätigen würde, dass stark auf Taube und/oder Wellensittich positive Seren immer auch auf Enten- und Gänsefedern reagieren. Dann würde sich für diese Seren sogar eine Analyse der Enten- und Gänsefedern-Antikörper erübrigen.

\section{Material und Methoden \\ $\nabla$}

\section{Zeitraum der Untersuchung}

Es wurden die Ergebnisse der IgG-Antikörper-Analysen von Entenfedern, Gänsefedern sowie Tauben- und Wellensittichfedern-, -kot und -serumextrakte vom 3.12.2013 bis zum 13.3. 2015 ausgewertet, die im Allergologisch-immunologischen Labor des Lungen- und Allergiezentrums des Malteser Lungenund Allergiezentrums Bonn durchgeführt worden waren. Die untersuchten Seren waren aus ganz Deutschland zur Antikörper-Diagnostik der exogen-allergischen Alveolitis eingesandt worden.
Technik der Antikörperuntersuchung und Normbereiche Die IgG-Antikörper wurden mit dem Phadia-ImmunoCAP-System (Fa. Thermo Fisher Scientific, Freiburg) mittels des Phadia 250 Gerätes analysiert. Der Hersteller empfiehlt in seiner Betriebsanleitung für den Vogel Taube einen Cut off von $10 \mathrm{mgA} / \mathrm{l}$, für Wellensittich $11 \mathrm{mgA} / \mathrm{l}$, für Entenfedern $10 \mathrm{mgA} / \mathrm{l}$ und Gänsefedern ebenfalls $10 \mathrm{mgA} / \mathrm{l}$.

Darüber hinaus hatten wir selbst Normgrenzen ermittelt. Dazu war für jedes Antigen das spezifische IgG im Serum von 20 gesunden Personen im Alter von 10 bis 75 Jahren (Durchschnittsalter 44 Jahre) bestimmt worden. Diese Personen waren Vogelstäuben und Schimmel nicht über das normale Maß hinaus exponiert gewesen und hatten nicht an Erkrankungen der Lunge, Allergien, Tumoren oder chronischer Entzündung gelitten. Da bekannt ist, dass die IgG-Antikörper gegen die EAA-Antigene nicht in einer Gauß'schen Verteilungskurve normalverteilt sind [15], wurde die 95\%-Perzentile als Cut-off festgelegt. Für das Antigen Taube hatte sich eine Normgrenze von $16 \mathrm{mg} / \mathrm{l}$, für Wellensittich von $7 \mathrm{mg} / \mathrm{l}$, für Entenfedern $9 \mathrm{mgA} / \mathrm{l}$ und für Gänsefedern von 11 $\mathrm{mgA} / \mathrm{l}$ ergeben [16].

\section{Graduierung der Antikörper}

Die quantitativ ermittelten positiven Werte wurden den üblichen vier Bereichen „fraglich positiv, schwach positiv, mittelstark positiv und stark positiv“ zugeordnet $[17,18]$. Die dafür verwendeten Grenzwerte dieser Bereiche wurden von unserem Labor selbst erstellt [16]. Die Bereiche zwischen den Grenzwerten für die Zuordnung der Antikörper sind in $\bullet$ Tab. 1 dargestellt.

\section{Auswertung der Antikörperergebnisse}

Die Häufigkeiten der IgG-Antikörper gegen Tauben- und Wellensittich-Antigene wurden aus 200 EAA-Suchtests in dem oben angegebenen Zeitraum ermittelt. Der IgG-Antikörper-Suchtest unseres Labors enthält die von uns aus der Literatur als die wichtigsten ermittelten und in den Seren unserer Patienten am häufigsten reagierenden Antigene der exogen-allergischen Alveolitis (๑ Tab.2) [19].

Zur Ermittlung der Häufigkeiten der IgG-Antikörper gegen Taube, Wellensittich, Ente und Gans wurden 200 Antikörperanalysen in der zeitlichen Reihenfolge ihrer Analyse, beginnend mit dem Datum des 13.12.2013, ausgewertet.

Tab. 2 Die Antigene des IgG-Antikörper-Suchtests auf exogen-allergische Alveolitis (EAA-Suchtest).

Taubenfedern-, -kot und -serumextrakte

Wellensittichfedern-, -kot und -serumextrakte

Saccharopolyspora rectivirgula (Micropolyspora faeni)

Thermoactinomyces vulgaris

Aspergillus fumigatus

Penicillium chrysogenum (notatum)

Aureobasidium pullulans (Pullularia pullulans)

Cephalosporium acremonium (Acremonium kiliense) 
Für die Fragestellung, wie häufig positive Enten- und Gänsefedern-Antikörper in demselben Serum von Tauben- oder Wellensittich-Antikörpern begleitet werden, standen in dem obengenannten Zeitraum die Untersuchungsergebnisse von 100 Patienten zur Verfügung, welche auf alle vier Antigene untersucht worden waren und auf Enten- oder/und Gänsefedern positiv reagiert hatten. Es wurden nur solche Ergebnisse berücksichtigt, zwischen denen der zeitliche Abstand der Analysen unter einem Monat lag.

Für die Fragestellung, ob Seren mit stark positiven Tauben- oder/ und Wellensittich-Antikörpern regelmäßig auch Enten- und Gänsefedernantikörper enthalten, standen die Untersuchungsergebnisse von 180 Seren zur Verfügung, welche auf Taube, Wellensittich, Ente und Gans untersucht worden waren (maximaler Abstand der einzelnen Analysen vier Wochen). Dabei wurden im Gegensatz zum vorausgehenden Kollektiv auch die Seren erfasst, welche auf Ente und Gans negativ reagiert hatten.

\section{Ergebnisse \\ $\nabla$}

IgG-Antikörper gegen Taube und Wellensittich im EAA-Suchtest

Im EAA-Suchtest (Taube, Wellensittich, Saccharopolyspora rectivirgula, Thermoactinomyces vulgaris, Aspergillus fumigatus, Penicillium chrysogenum, Aureobasidium pullulans und Cephalosporium acremonium [Acremonium kiliense]) waren in $200 \mathrm{Se}-$ ren $67 \%$ der Seren (134 Seren) sowohl auf Taube als auch auf Wellensittich negativ bei Verwendung der von uns ermittelten Cut-off-Werte ( Abb.1). Auf Taube und auf Wellensittich zugleich reagierten 16\% der Seren (32 Seren), nur auf Taube 15,5\% (31 Seren) und nur auf Wellensittich 1,5\% (3 Seren) ( $\bullet$ Abb. 1).

Häufigkeiten der Antikörper gegen Taube, Wellensittich, Ente und Gans

In $\odot$ Abb. 2 sind die Häufigkeiten der Antikörper gegen Taube, Wellensittich, Ente und Gans unabhängig davon, ob in demselben Serum weitere Antikörper vorkommen, grafisch dargestellt. Es ist zu erkennen, dass die Antikörper gegen Tauben-Antigene deutlich am häufigsten vorkommen und Entenfedern-Antikörper häufiger als Gänsefedern-Antikörper sind.

\section{Gemeinsamens Vorkommen von Antikörpern gegen Ente und Gans mit Taube und Wellensittich}

Die Auswertung der 100 Seren mit positiven Enten- oder/und Gänsefedern-Antikörpern, die auch auf Taube und Wellensittich untersucht worden waren, ergab, dass alle Seren bis auf zwei Seren mit fraglich positiven Antikörpern gegen Entenfedern auch auf Taube oder/und Wellensittich reagierten. Wenn nach den Normgrenzen (Cut-off-Werte) des Herstellers Phadia ausgewertet wird, so ist sogar nur ein einziges Serum (mit einem fraglich positiven Antikörper gegen Entenfedern) auf Taube und Wellensittich negativ. Somit kommen eindeutig positive BettfedernAntikörper nie isoliert, sondern nur zusammen mit Tauben- und Wellensittich-Antikörpern vor.

Dies bedeutet aus der Sicht der Tauben- und Wellensittich-Antikörper, dass bei deren Fehlen auch keine eindeutig positiven Bettfedern-Antikörper vorkommen ( $\bullet$ Abb.3).

In $\triangle$ Abb. 3 sind die quantitativen Untersuchungsergebnisse anschaulich dargestellt. Es ist zu erkennen, dass die Tauben- und Wellensittich-Antikörper häufiger stark positiv sind als die Enten- und Gänsefedern-Antikörper.

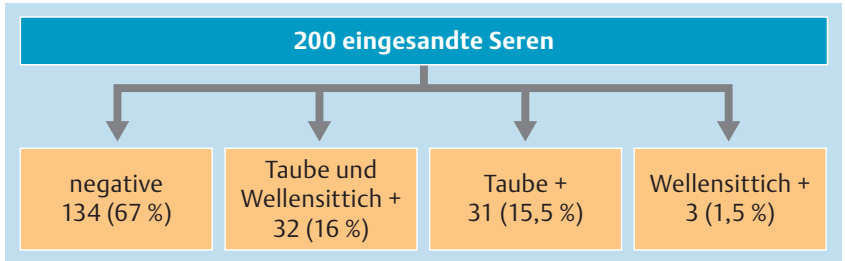

Abb.1 Antikörper gegen Taube und Wellensittich in 200 eingesandten Seren.

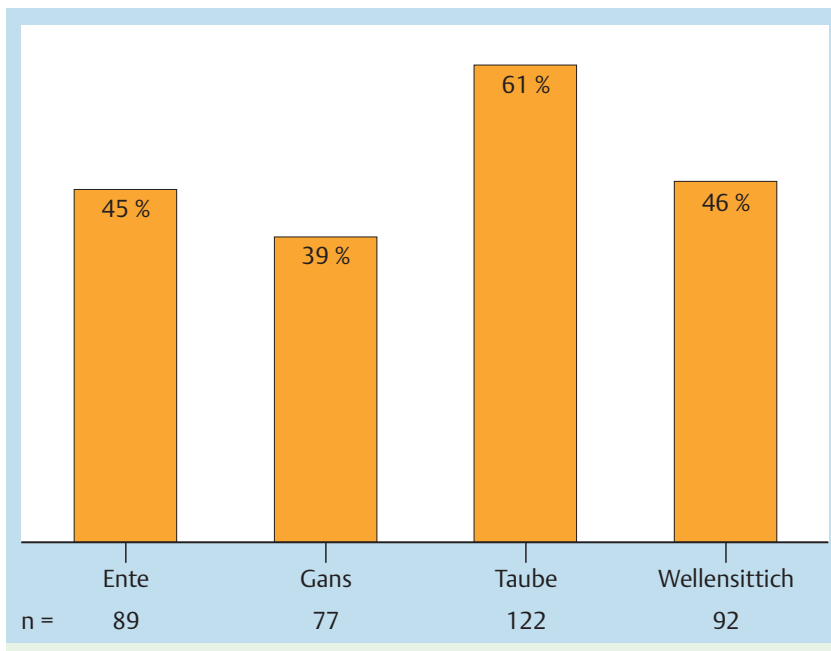

Abb. 2 Häufigkeiten von IgG-Antikörpern gegen Taube, Wellensittich, Ente und Gans in 200 Seren.

Regelmäßiges Vorkommen von Bettfedern-Antikörpern in Seren mit stark positiven Tauben- oder/und Wellensittich-Antikörpern

Aus $\bullet$ Abb. 4 geht hervor, dass die Bettfedern-Antikörper in Seren mit stark positiven Tauben- und Wellensittich-Antikörpern häufiger vorkommen als in Seren mit schwach positiven Vogel-Antikörpern. Alle stark positiven Tauben-Antikörper ( $\bullet$ Tab.1) sind mit Enten- und/oder Gänsefedern-Antikörpern assoziiert. Auch die mittelstark positiven Tauben-Antikörper oberhalb der in - Abb.4 eingezeichneten Grenze von $84 \mathrm{mgA} / \mathrm{l}$ sind mit wenigstens einem Bettfedern-Antikörper (Ente oder Gans) assoziiert.

Auch alle Seren mit stark positiven Wellensittich-Antikörpern ( $\odot$ Tab. 1) enthalten in $\odot$ Abb. 4 auch Bettfedern-Antikörper sowie die mittelstark positiven Seren oberhalb der in 0 Abb. 4 eingezeichneten Grenze bei $103 \mathrm{mgA} / \mathrm{l}$.

Die Anzahl der Seren oberhalb der in 0 Abb. 4 eingezeichneten Grenzen beträgt für die Taube 27 und für den Wellensittich 24 . Bezogen auf die Gesamtzahl der 180 ausgewerteten Seren sind das $15 \%$ der Seren. Für den Wellensittich sind es 13\% der Seren in dem Bereich oberhalb der Grenze von $103 \mathrm{mgA} / 1$. Im Durchschnitt liegen somit $14 \%$ der Seren in einem Bereich hoher Vogel-IgG-Antikörper, die ausnahmslos von Bettfedern-Antikörper begleitet werden.

Somit kommt den quantitativ bestimmten IgG-Antikörpern gegen Taube und Wellensittich nicht nur die diagnostische Bedeutung zu, bei 67 von 100 Seren (67\%) neben einer Vogelhalterlunge von Taube und Wellensittich auch eine Bettfedern-Alveolitis auszuschließen, sondern darüber hinaus bei $14 \%$ der verbliebenen 33 Seren, das sind 4 Seren, indirekt Bettfedern-Antikörper anzuzeigen. Addiert man diese 67 und die 4 Seren, so erhält man die 


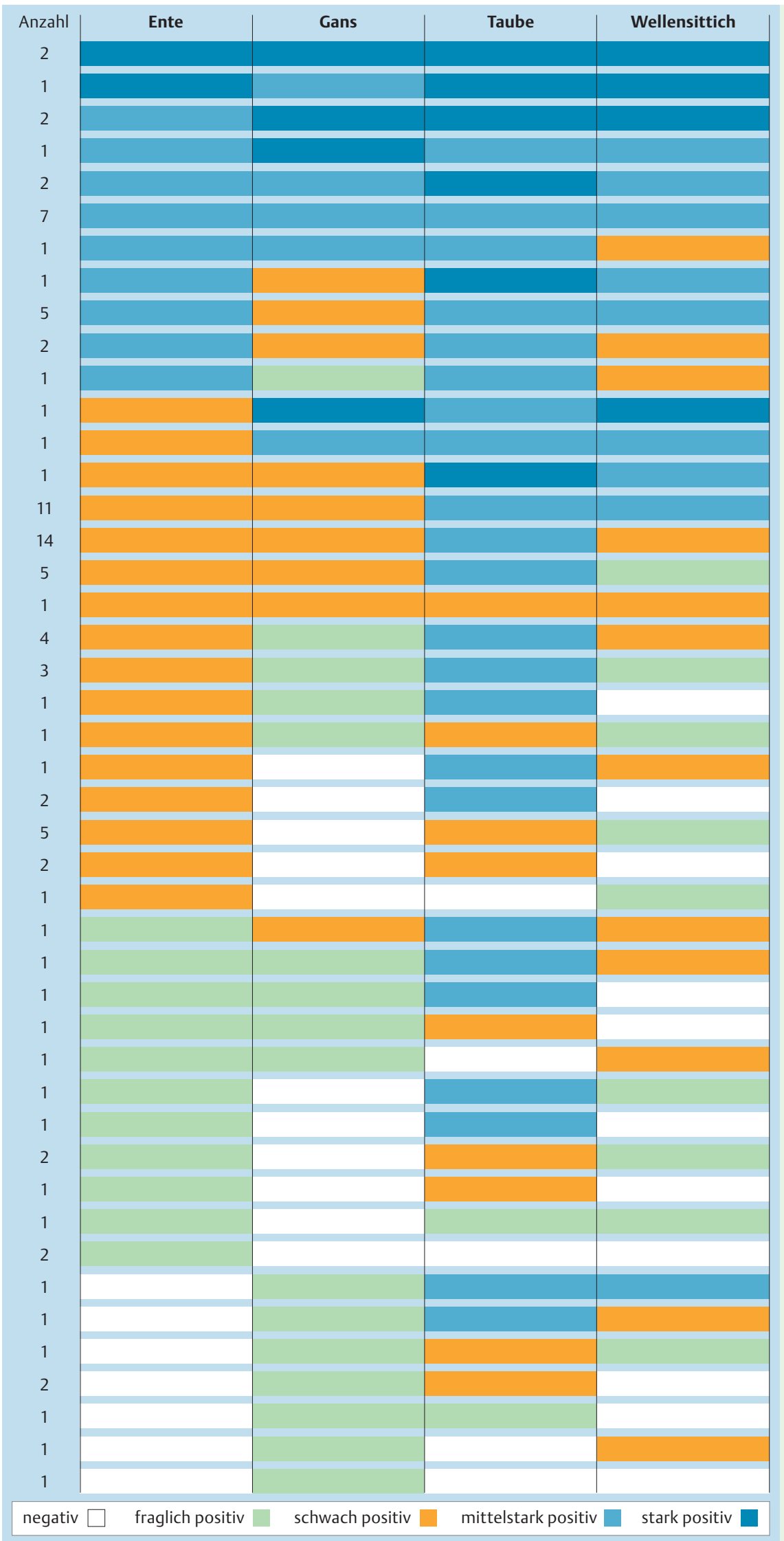

Abb.3 Die Reaktionsmuster der verschiedenen Antikörperstärken der 100 auf Taube, Wellensittich, Ente und Gans untersuchten Seren.

Summe von 71 von 100 Seren (71\%), bei denen die Tauben- und Wellensittich-Antikörper die Bettfedern-Antikörper ausgeschlossen oder indirekt detektiert haben.
Das bedeutet, dass bei Berücksichtigung der Tauben- und Wellensittich-Antikörper, die im EAA-Suchtest anfallen, nur noch von 100 Seren 29 auf Enten- und Gänsefedern-Antikörper untersucht zu werden brauchen. 


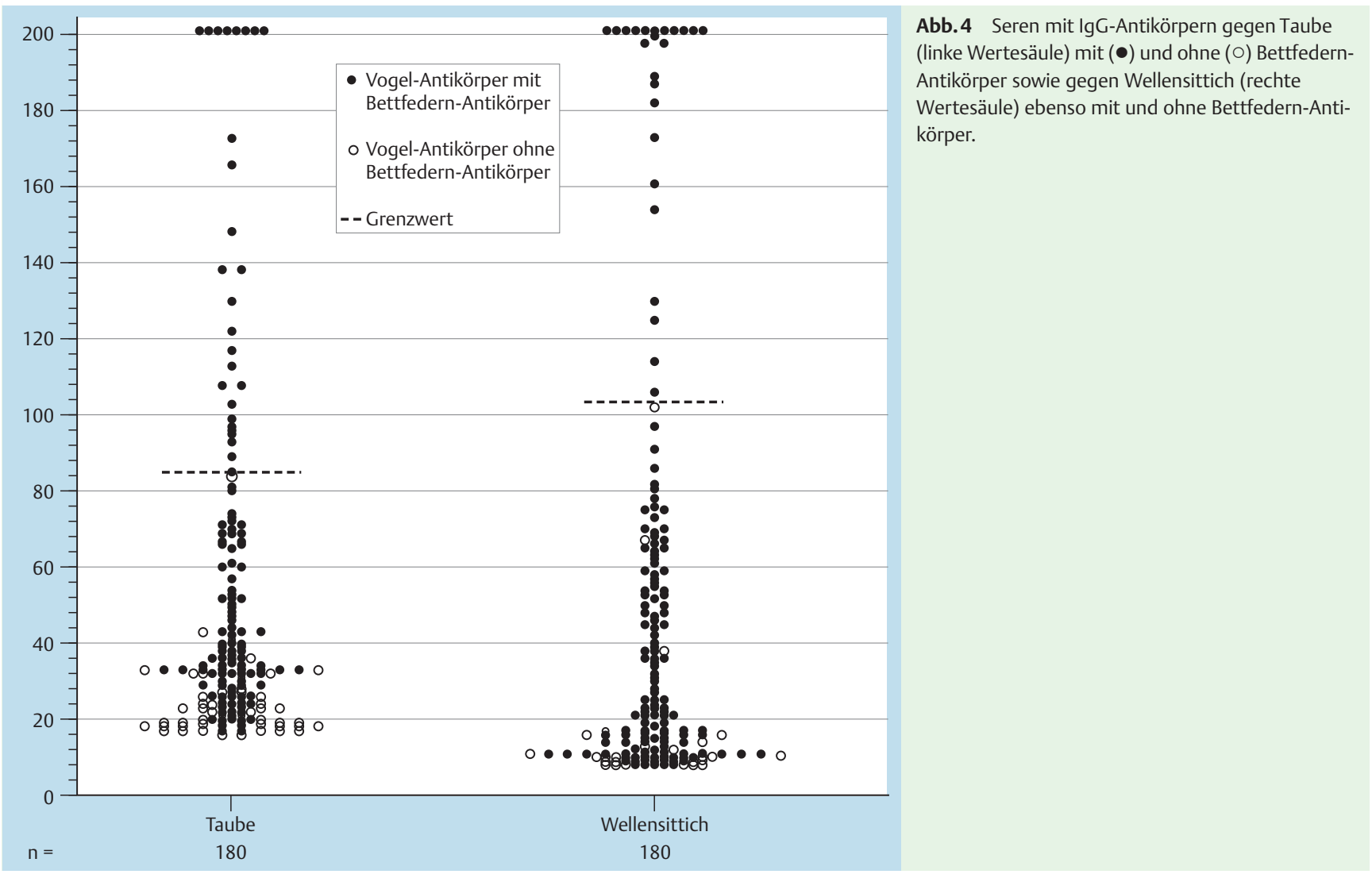

Basierend auf diesen Ergebnissen schlagen wir folgendes diagnostisches Procedere zur Erkennung der wichtigsten FedernAntikörper der EAA vor:

1. Schritt: Bei Verdacht auf eine EAA werden im Rahmen des EAA-Suchtests alle Seren auf die Tauben- und WellensittichAntigene untersucht. Mit dieser Testkombination werden bei Anwendung der Normgrenzen des Testherstellers auch die Bettfedern-Antikörper (Enten- und Gänsefedern) erfasst.

2. Schritt: Bei IgG-Antikörperwerten gegen Tauben-Antigene oberhalb von $84 \mathrm{mgA} / \mathrm{l}$ und Wellensittich-Antigene oberhalb von $103 \mathrm{mgA} / \mathrm{l}$ wird dem behandelnden Arzt mitgeteilt, dass der Patient nicht nur auf Taube oder/und Wellensittich sensibilisiert ist, sondern auch auf Daunenfedern von Ente oder/ und Gans.

3. Schritt: Bei Werten darunter wird dem behandelnden Arzt, der die Antikörperdiagnostik veranlasst hat, empfohlen, eine zusätzliche Analyse auf Enten- und Gänsefedern-Antikörper zu veranlassen.

4. Schritt: In Rahmen einer der nächsten Testansätze werden nur noch die Seren auf Enten- oder/und Gänsefedern-Antikörper auf Veranlassung des zuweisenden Arztes untersucht, bei denen die erste Untersuchung Bettfedern-Antikörper weder ausgeschlossen noch indirekt nachgewiesen hatte.

\section{Diskussion \\ $\nabla$}

Der nicht unbeträchtliche Prozentsatz positiver Seren auf Tauben- und Wellensittich-IgG-Antikörper im EAA-Suchtest von $33 \%$ Abb. 1) zeigt, dass diese Vögel in hohem Maße sensibilisieren und dass die Einsender eine spezifische Auswahl bei ihren Patienten getroffen haben. In der Normalbevölkerung kommen diese IgG-Antikörper nur bei $1 \%-15 \%$ je nach Messmethode vor [19].

Nach bisheriger Kenntnis sind Tauben und Sittiche die weitaus häufigsten Verursacher der exogen-allergischen Alveolitis in Form der Vogelhalterlunge [19-21]. In der oben erwähnten spanischen Studie von Morell waren bei der chronischen fibrosierenden Verlaufsform hingegen die Bettfedern die häufigste Krankheitsursache [6].

Unsere Auswertung der Tauben- und Wellensittich-Antigene auf der einen Seite und der Bettfedern-Antigene auf der anderen Seite zeigt, dass die uns eingesandten Seren am häufigsten Antikörper gegen Tauben- und Wellensittichfedern enthalten ( $\bullet$ Abb.2). Auch die Antikörperkonzentrationen sind bei der Taube und beim Wellensittich am höchsten ( $\bullet$ Abb. 3).

Nach einer Studie von Koschel liegen die Enten- und Gänsefedern-Antikörper bei der Bettfedern-Alveolitis höher als bei der Taubenzüchterlunge und der Wellensittichhalterlunge [3].

Bei der Interpretation unserer Ergebnisse ist zu berücksichtigen, dass uns häufig Seren eingesandt werden, wenn die Patienten angeben, Kontakt mit Tauben oder Sittichen zu haben; dass aber nach unserer Erfahrung seltener Seren auf Bettfedern-Antikörper eingesandt werden, wenn Patienten in Federbetten schlafen. Insofern lassen die Frequenzen der IgG-Antikörper gegen diese aviären Antigene keine Rückschlüsse darauf zu, wie häufig diese Antikörper in der Bevölkerung vorkommen und erst recht nicht, wie häufig die Vogelhalterlunge im Vergleich zur Bettfedern-Alveolitis ist. Auch kann man den Abbildungen nicht entnehmen, mit welchen der vier Antigene die Patienten inhalativen Kontakt hatten und welche Antikörper von Kreuzreaktionen hervorgerufen wurden. Man erkennt aber, dass die zahlreichen Enten- und Gänsedaunen-Antikörper ein nicht zu vernachlässigendes Krankheitspotenzial in Deutschland darstellen. 


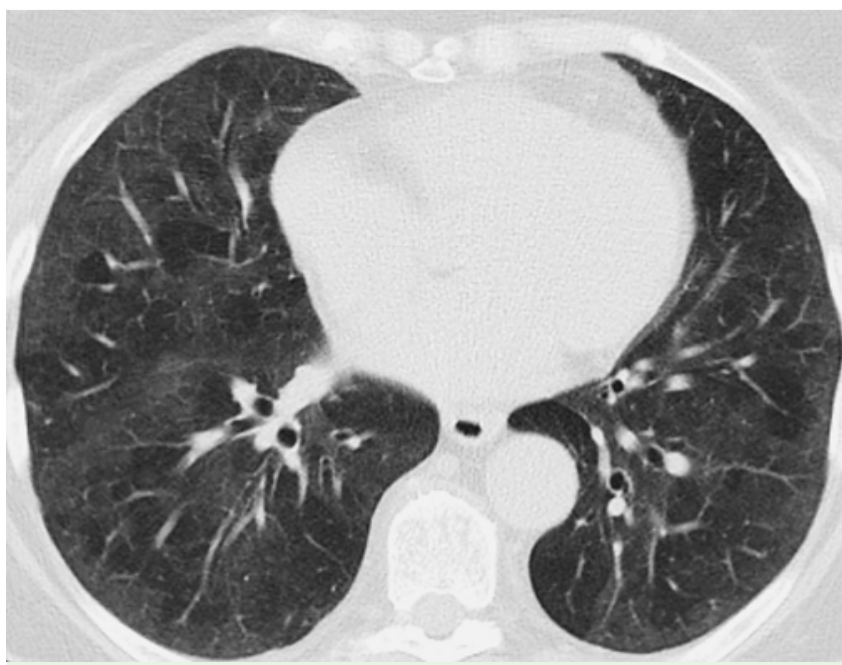

Abb.5 HRCT-Schichtlunge einer Patientin mit Wellensittichhalterlunge und Bettfedern-EAA. Die typische Milchglastrübung und lokale Überblähungsareale sind gut zu erkennen. (Ich danke der Praxis für Radiologie Kaiserpassage Bonn für die Aufnahme.)

Das wohl interessanteste und diagnostisch besonders relevante Ergebnis dieser Studie ist, dass eindeutig positive BettfedernAntikörper nicht isoliert, sondern immer nur zusammen mit Tauben- und Wellensittich-Antikörpern vorkommen. Die nur in $1 \%$ bzw. $2 \%$ (nach den Normwerten der Firma bzw. von uns selbst erhoben) vorkommenden fraglich positiven Enten- und GänseAntikörper ohne Tauben- und Wellensittich-Antikörper sind nur von marginaler klinischer Relevanz, da bei solchen fraglich positiven Vogel-Antikörpern (Taube) nur in $4 \%$ eine allergische Alveolitis vorliegt [22].

Die Normgrenzen für die Antikörper gegen Vogel-Antigene und andere EAA-Antigene in Deutschland werden zurzeit mit einer multizentrischen deutschen Studie evaluiert. Vorausichtlich wird man sich dann an den neuen Werten orientieren können.

Je höher die Tauben- oder Wellensittich-Antikörper liegen, desto wahrscheinlicher sind gleichzeitige Daunen-Antikörper in demselben Serum ( $\bullet$ Abb.4). Oberhalb der Grenzen von $84 \mathrm{mgA} / \mathrm{l}$ für Taube und von $103 \mathrm{mgA} / \mathrm{l}$ für Wellensittich erübrigt sich eine zusätzliche Bestimmung der Daunen-Antikörper, da alle Seren oberhalb der Grenzen auch Daunen-Antikörper enthalten.

Wenn unterhalb dieser Grenzen eine zusätzliche Antikörperbestimmung auf Enten- und Gänsefedern negativ ausgefallen ist, so ist daran zu denken, dass auch die nachgewiesenen Taubenoder Wellensittich-Antikörper eine klinische Relevanz haben können. Denn die Federnpartikel dieser Vögel können, manchmal auf den ersten Blick nicht erkennbar, von wilden Tauben auf Fensterbänken herrühren oder in Teppichen oder Kleidung vorkommen, die früher Sittichen ausgesetzt waren [23-24].

Die oben beschriebene serologische Stufendiagnostik hat gegenüber dem bisherigen diagnostischen Vorgehen zwei Vorteile. Einmal wird mit dem EAA-Suchtest unabhängig von der Anamnese (die in Bezug auf Vogelstäube nicht vollständig sein kann) bei diesen ubiquitären aviären Antigenen ein größerer Kreis von Patienten mit interstitiellen Lungenkrankheiten erfasst, was bei der großen Dunkelziffer von heute unerkannten EAAs dringend angezeigt ist [6]. Und andererseits werden Kosten gespart, weil durch die Vortestung durch den ohnehin erforderlichen EAASuchtest nur noch für 29\% der eingesandten Seren eine Antikörperbestimmung auf Enten- und Gänsefedern erforderlich ist.
Für das gleichzeitige Vorkommen von Enten- und GänsefedernAntikörpern in Seren mit stark positiven Tauben- und Wellensittich-Antikörpern sind wahrscheinlich Kreuzreaktionen, insbesondere mit den Albuminen in den Federn, im Kot und Vogelserum verantwortlich $[19,25]$. Diese Antikörper gegen kreuzreagierende Vogel-Albumine wurden gehäuft bei Patienten mit einer Vogelhalterlunge und in Seren mit hochkonzentrierten IgG-Antikörpern gegen Vogel-Antigenextrakte gefunden [26]. In Seren mit weniger stark positiven Antikörpern kommen die Albumin-Antikörper seltener vor [26]; das mag der Grund dafür sein, dass bei den schwächer positiven Antikörpern weniger Übereinstimmungen bei den Tauben-, Wellensittich-, Entenund Gänse-Antikörpern zu finden sind. Die Antigen-Epitope der niedertitrigen IgG-Antikörper sind speziesspezifisch [19].

Eine vergleichbare andere Studie zu diesem Thema einer Detektion der Bettfedern-Antikörper über Tauben- und Sittich-Antikörper ist uns nicht bekannt.

Bei Patienten, die angeben, keinen Kontakt mit Ziervögeln und Tauben zu haben, nur die Antikörper gegen Enten- und Gänsefedern bestimmen zu lassen, ist unseres Erachtens nicht ratsam. Denn Taubenantigen (Federn, Kot) als häufigstes EAA-Antigen kommt auf Fensterbänken, an Arbeitsplätzen, über Klimaanlagen verteilt in Geschäften und Büros als hochpotentes Antigen ubiquitär vor. Das gilt auch für Sittichallergene, z.B. in Teppichen von Vormietern [23] und in der Kleidung von Kontaktpersonen [24], was anamnestisch meist primär nicht zu erfassen ist.

Wichtig zu wissen ist, dass wegen der Kreuzreaktionen von lebenden Vögeln und den Daunen bei der Taubenzüchter- und anderen Vogelhalterlungen die Daunenfedern in Kissen und in Kleidung eine zusätzliche Allergenbelastung darstellen. Deshalb sind bei der Therapie der Vogelhalterlunge nicht nur die lebenden Vögel, sondern auch die Daunen zu meiden. Sicherlich ist für manchen progredienten Verlauf einer Vogelhalterlunge trotz Vogel-Karenz dieser Daunenkontakt verantwortlich [27].

Je höher ein Antikörper gegen Vögel oder Bettfedern liegt, desto wahrscheinlicher leidet der Patient an allergischer Alveolitis [22, 27].

- Abb.5 zeigt einen typischen Röntgenbefund einer Patientin mit einer Wellensittichhalterlunge, bei der die IgG-Antikörperkonzentration des Wellensittich-Antikörpers mit $818 \mathrm{mgA} / \mathrm{l} \mathrm{sehr}$ hoch lag.

Bei der Bettfedern-Alveolitis kann eine Verdachtsdiagnose sehr gut durch einen Karenz-Versuch (Entfernen der Federn-Oberbetten und Federn-Kopfkissen) und ggf. zusätzlich einen Reexpositionstest (Provokation) in Form von ,einer Nacht wieder im alten Federbett schlafen' bestätigt werden $[1,2,4,6,11]$.

Vereinzelt wurden Patienten mit Vogelhalterlunge ohne nachweisbare Antikörper (seronegative Vogelhalterlunge) beschrieben $[23,28]$. Eine seronegative Bettfedern-Alveolitis ist hingegen bisher nicht bekannt.

\section{Danksagung}

Andreas Sennekamp danken wir für die englische Übersetzung der Zusammenfassung.

\section{Interessenkonflikt}

$\nabla$

Die Autoren geben an, dass kein Interessenkonflikt besteht. 


\section{Literatur}

1 Kroidl R, Wieshammer S, Sennekamp J. Bettfedern-Alveolitis: 4 besondere Fälle. Allergologie 2003; 26: 334-339

2 Inase N, Ohtani Y, Sumi Y et al. A clinical study of hypersensitivity pneumonitis presumably caused by feather duvets. Ann Allergy Asthma Immunol 2006; 96: 98-104

3 Koschel D, Lützkendorf L, Wiedemann B et al. Antigen-specific IgG antibodies in feather duvet lung. Eur J Clin Invest 2010; 40: 797-802

4 Koschel $D$, Wittstruck $H$, Renck $T$ et al. Presenting features of feather duvet lung. Int Arch Allergy Immunol 2010; 152: 264-270

5 Koschel D. Die chronische EAA - ein häufig übersehenes Krankheitsbild im Schatten des „IPF-Hype“. Pneumologe 2015; 12: 59-61

6 Morell F, Villar A, Montero MA et al. Chronic hypersensitivity pneumonitis in patients diagnosed with idiopathic pulmonary fibrosis: a prospective case-cohort study. Lancet Respir Med 2013; 1: 685-694

7 Plessner MM. Une maladie des trieurs de plumes: la fièvre de canard. Arch Mal Prof 1960; 21: 67-69

8 Wettengel R In: Hamm J: Vogelhalterlunge - allergische Alveolitis und Lungenfibrosen durch Antigeninhalation. Interstitielle Lungenerkrankungen, Lungenfibrosen. Tuberkulose-Bücherei Stuttgart: Thieme; 1975: $36-44$

9 Schiele R, Lutgen W. Berufsbedingte allergische Alveolitis beim Umgang mit Bettfedern. Arbeitsmed Sozialmed Präventivmed 1978; 13: $36-39$

10 Burdon JGW, Stone C. Bird fancier's lung after an unusual exposure to avian protein. Amer Rev Respir Dis 1986; 134: 1319-1320

11 Sennekamp J. Exogen-allergische Alveolitis. Monographie München: Dustri-Verlag; 1998

12 Joest $M$, Schulte $W$, Sennekamp J. Bedeutung eines breiten Spektrums spezifischer IgG-Antikörper für die Diagnose einer exogen-allergischen Alveolitis. Allergologie 2014; 37: 220-224

13 Eade OE, Hodges JR, Berrill WT et al. Immunfluorescent antibodies in patients with bird fancier's lung. Clin Exp Immunol 1978; 32: 259262

14 Sennekamp J, Lange $G$, Nerger $K$ et al. Human antibodies against antigens of the sparrow, blackbird, weaver finch, canary, budgerigar, pigeon and hen using the indirect immunofluorescent technique. Clin Allergy 1981; 11: $375-384$

15 Bañales JL, Vazquez L, Mendoza F et al. On the correct determination of reference values for serum antibodies against pigeon serum antigen using a group of healthy blood donors. Arch Med Res 1997; 28: 289 291

16 Sennekamp J, Lehmann E, Joest M. Die berufsbedingte exogen-allergische Alveolitis. Arbeitsmedizin, Sozialmedizin, Umweltmedizin 2015; 50: $38-52$

17 Sennekamp J, Baur X, Jaeger D et al. Standardisierung von IgG-Antikörpern der exogen-allergischen Alveolitis. Allergologie 1992; 15: 23

18 Sennekamp J. Standardisierung der Antikörperbefunde der exogenallergischen Alveolitis. Allergologie 2000; 23: 45-47

19 Sennekamp J. Extrinsic allergic alveolitis - hypersensitivity pneumonitis. Monographie München: Dustri; 2004

20 Hendrick DJ, Faux JA, Marshall R. Budgerigar fancier's lung: the commonest variety of allergic alveolitis in Britain. Brit Med J 1978; II: 81 84

21 Müller S, Müller N. Exogen-allergische Alveolitiden - 10-jährige Leistungsbilanz eines Referenzzentrums in der DDR. Z Klin Med 1986; 41: $85-88$

22 Banham SW, McSharry CP, Lynch PP et al. Relationship between avian exposure, humoral immune response, and pigeon breeder's disease among Scottish pigeon fanciers. Thorax 1986; 41: 274-278

23 Greinert U, Lepp U, Becker WW. Bird keeper's lung without bird keeping. Eur J Med Res 2000; 5: 124

24 Merget $R$, Sander I, Ewig $S$ et al. Consort hypersensitivity pneumonitis. Eur Respir J 2009; 33: 1 -4

25 Tauer I, Baur X, Fruhmann G et al. Ursächliche Antigene der Vogelhalterlunge. Atemw-Lungenkrkh 1989; 15: 226-227

26 Sennekamp J, Stroehmann I, Geisler LS et al. Klinische Relevanz immunserologischer Untersuchungen bei Taubenzüchterlunge. Pneumologie 1975; 152: $51-55$

27 Wittmann M, Petro P. Zur Progredienz der Vogelhalterlunge durch Bettfedern. Atemw Lungenkrkh 2000; 26: 581-585

28 Sennekamp J, Niese D, Stroehmann I et al. Pigeon breeder's lung lacking detectable antibodies. Clin Allergy 1978; 8: 305-310 